PROCEEDINGS OF THE

AMERICAN MATHEMATICAL SOCIETY

Volume 134, Number 12, December 2006, Pages 3613-3624

S 0002-9939(06)08423-1

Article electronically published on June 12, 2006

\title{
ALMOST AUTOMORPHIC SOLUTIONS FOR SEMILINEAR BOUNDARY DIFFERENTIAL EQUATIONS
}

\author{
S. BOULITE, L. MANIAR, AND G. M. N'GUÉRÉKATA \\ (Communicated by Carmen C. Chicone)
}

\begin{abstract}
In this work, we use the extrapolation methods to study the existence and uniqueness of almost automorphic solutions to the semilinear boundary differential equation

$$
(S B D E)\left\{\begin{array}{l}
x^{\prime}(t)=A_{m} x(t)+h(t, x(t)), t \in \mathbb{R} \\
L x(t)=g(t, x(t)), t \in \mathbb{R}
\end{array}\right.
$$

where $A:=A_{m} \mid$ ker $L$ generates a hyperbolic $C_{0}$-semigroup on a Banach space $X$ and $h, g$ are almost automorphic functions which take values in $X$ and a "boundary space" $\partial X$, respectively. These equations are an abstract formulation of partial differential equations with semilinear terms at the boundary, such as population equations, retarded differential equations and boundary control systems. An application to retarded differential equations is given.
\end{abstract}

\section{INTRODUCTION}

We are concerned with the existence and the almost automorphy of solutions of the semilinear boundary differential equation

$$
(S B D E)\left\{\begin{array}{l}
x^{\prime}(t)=A_{m} x(t)+h(t, x(t)), t \in \mathbb{R}, \\
L x(t)=g(t, x(t)), t \in \mathbb{R} .
\end{array}\right.
$$

The first equation stands in a Banach space $X$ called state space, and the second in a "boundary space" $\partial X ;\left(A_{m}, D\left(A_{m}\right)\right)$ is a densely defined linear operator on $X$, and $L: D\left(A_{m}\right) \longrightarrow \partial X$ is bounded linear, $h: \mathbb{R} \times X \longrightarrow X$ and $g: \mathbb{R} \times X \longrightarrow \partial X$. This kind of equation is motivated by retarded differential equations in continuous functions spaces, by dynamic population equations in $L^{1}$-space with semilinear birth processes, and by boundary control problems.

We assume that the operator $A:=A_{m} \mid \operatorname{ker} L$ generates a hyperbolic $C_{0^{-}}$-semigroup on $X$ and $h(\cdot, x), g(\cdot, x)$ are almost automorphic functions on $\mathbb{R}$ for each $x$ in $X$ and globally Lipschitzian. Under additional assumptions, called Greiner assumptions (see Section 4), on the boundary operator $L$, we show that there is a unique almost automorphic mild solution to (SBDE) which satisfies a variation of constant formula.

Received by the editors June 13, 2005 and, in revised form, July 6, 2005.

2000 Mathematics Subject Classification. Primary 34A05, 34G20,47A55.

Key words and phrases. Almost automorphic functions, semilinear boundary differential equations, retarded differential equations, hyperbolic semigroups, extrapolation space, Dirichlet map. 
The idea to achieve this aim is to transform the boundary equation (SBDE) (see Section 4) into an equivalent semilinear evolution equation as follows:

$$
x^{\prime}(t)=A_{-1} x(t)+h(t, x(t))+\left(\lambda-A_{-1}\right) L_{\lambda} g(t, x(t)), \quad t \in \mathbb{R},
$$

where $A_{-1}$ is the continuous extension of $A$ to the extrapolated Banach space $X_{-1}$ of $X$ with respect to $A$. Under the Greiner assumptions on $L$, the operator $L_{\lambda}:=\left(L \mid \operatorname{ker}\left(\lambda-A_{m}\right)\right)^{-1}$, called the Dirichlet map of $A_{m}$, is bounded from $\partial X$ to $X$, and the semilinear term $f(t, x):=h(t, x)+\left(\lambda-A_{-1}\right) L_{\lambda} g(t, x)$ is an $F_{A_{-1}}$-valued function, where $F_{A_{-1}}$ is the Favard class of $A_{-1}$, which is a larger Banach space than $X$. Although this equation is written in $X_{-1}$, the task is to show the existence of mild solutions in $X$. For the mentioned notions, see Section 2 for definitions and [9] for more details. The extrapolation theory has been introduced by Da Prato and Grisvard [6] and Nagel [9] and used for various purposes; see [1, 2, 3, 4, 15, 17, 21.

According to this transformation, we begin in Section 3 by studying the existence of almost automorphic solutions of the semilinear evolution equation

$$
(S E E) \quad x^{\prime}(t)=A_{-1} x(t)+f(t, x(t)), \quad t \in \mathbb{R},
$$

where $A_{-1}$ is the extrapolation of a generator $A$ of a hyperbolic $C_{0}$-semigroup $(T(t))_{t>0}$ on a Banach space $X$. The semilinear term $f$ is defined on $\mathbb{R} \times X$ with values in the extrapolated Favard class $F_{A_{-1}}$. We show first that the inhomogeneous evolution equation

$$
(I E E) \quad x^{\prime}(t)=A_{-1} x(t)+g(t), \quad t \in \mathbb{R},
$$

has a unique almost automorphic mild solution on $X$ for each almost automorphic function $g: \mathbb{R} \longrightarrow F_{A_{-1}}$, and this yields by the above equivalence the existence of almost automorphic solutions of the inhomogeneous boundary differential equation

$$
(I B D E)\left\{\begin{array}{l}
x^{\prime}(t)=A_{m} x(t)+h_{1}(t), \quad t \in \mathbb{R}, \\
L x(t)=h_{2}(t), \quad t \in \mathbb{R} .
\end{array}\right.
$$

The contraction fixed point theorem then yields the unique almost automorphic mild solution on $X$ for the semilinear evolution equation (SEE), and thus the one of the boundary differential equation (SBDE).

In the particular case where $g=0$ and $h_{2}=0$, the boundary equations (SBDE) and (IBDE) become

$$
x^{\prime}(t)=A x(t)+h(t, x(t)), \quad t \in \mathbb{R}, \quad x^{\prime}(t)=A x(t)+h_{1}(t), \quad t \in \mathbb{R},
$$

evolution equations on $X$, which are much considered in the literature. The almost automorphy of such equations was studied in [8, 10, 13, 18, 19, 20], where the exponential stability of $(T(t))_{t \geq 0}$ is required. Recently, the hyperbolic case was treated by the authors in [5].

In the last section of this paper, we apply the abstract results to the retarded differential equation

$$
(R D E) \quad \frac{d}{d t} x(t)=B x(t)+f\left(t, x_{t}\right), \quad t \in \mathbb{R},
$$

where $B$ generates a $C_{0}$-semigroup $(S(t))_{t \geq 0}$ on a Banach space $E$ and $f$ is a nonlinear function from $\mathbb{R} \times C([-r, 0], E)$ into $E$. Under some assumptions on $B$, we show that the function-solution $t \longmapsto x_{t}$ is almost automorphic in $C([-r, 0], E)$, and then $x(\cdot)$ is almost automorphic in $X$. 
Actually, the problem of finding an almost automorphic solution to inhomogeneous differential equations goes back to S. Bochner and W. A. Veech, and has attracted many mathematicians (see [14, [18, [20] and [22] for exhaustive lists of references). In 14, for instance, Russell A. Johnson has shown that the almost periodic ODE $x^{\prime}+A(t) x=B(t)$ admits no almost periodic solution. He constructed an interesting example of a two-dimensional almost periodic system whose projective flow has an almost automorphic minimal subset which is not almost periodic. He also proved that some equation in the hull of the above equation admits an almost automorphic solution which is not almost periodic.

\section{Preliminaries}

We begin in this section by fixing some notations and recalling a few basic results on extrapolation spaces of generators. For more details, we refer the reader to [9] and [17. Let $(A, D(A))$ be the generator of a $C_{0}$-semigroup $(T(t))_{t \geq 0}$ on a Banach space $X$.

Define on $X$ a new norm by

$$
\|x\|_{-1}=\left\|(\lambda-A)^{-1} x\right\|, \quad x \in X, \quad \lambda \in \rho(A) .
$$

The completion of $\left(X,\|\cdot\|_{-1}\right)$ is called the extrapolation space of $X$ associated to $A$ and will be denoted by $X_{-1}$. By the resolvent equation, the space $X_{-1}$ does not depend on $\lambda$.

Since $T(t)$ commutes with the operator resolvent $R(\lambda, A):=(\lambda I-A)^{-1}$, the extension of $T(t)$ to $X_{-1}$ exists and defines a $C_{0}$-semigroup $\left(T_{-1}(t)\right)_{t \geq 0}$ which is generated by $A_{-1}$ with $D\left(A_{-1}\right)=X$.

We recall that the Favard class associated to a generator $A$ (or $T(\cdot))$ is the Banach space

$$
F_{A}:=\left\{x \in X: \sup _{t>0} \frac{1}{t}\left\|e^{-\omega t} T(t) x-x\right\|<\infty\right\}
$$

endowed with the norm

$$
\|x\|_{F_{A}}:=\sup _{t>0} \frac{1}{t}\left\|e^{-\omega t} T(t) x-x\right\| .
$$

Here $\omega>\omega_{0}(T(\cdot))$, the growth bound of $T(\cdot)$. We note that $F_{A}$ is independent of the choice of $\omega$, contains the domain of $A, F_{A} \hookrightarrow X \hookrightarrow F_{A_{-1}} \hookrightarrow X_{-1}$, and

$$
\left(\lambda-A_{-1}\right): F_{A} \longrightarrow F_{A_{-1}}
$$

is an isomorphism for every $\lambda \in \rho(A)$. In the case when $X$ is a reflexive Banach space, the Favard class associated to $T(\cdot)$ is exactly the domain of its generator (see, e.g., [9, Section. II.5.b] for more properties).

A $C_{0}$-semigroup $(T(t))_{t \geq 0}$ is said to be hyperbolic if it satisfies the following properties:

(i) there exist two subspaces $X^{S}$ (the stable space) and $X^{U}$ (the unstable space) of $X$ such that $X=X^{S} \oplus X^{U}$;

(ii) $T(t)$ is defined on $X^{U}, T(t) X^{U} \subset X^{U}$, and $T(t) X^{S} \subset X^{S}$ for all $t \geq 0$;

(iii) there exist constants $M, \delta>0$ such that

$$
\left\|T(t) P_{S}\right\| \leq M e^{-\delta t}, \quad t \geq 0, \quad\left\|T(t) P_{U}\right\| \leq M e^{\delta t}, \quad t \leq 0,
$$

where $P_{S}$ and $P_{U}$ are, respectively, the projections onto $X^{S}$ and $X^{U}$. 
In the sequel we need the following main proposition.

Proposition $2.1([2])$. Assume that the semigroup $T(\cdot)$ is hyperbolic. Then:

(i) $P_{S} T(t)=T(t) P_{S}$ and $P_{U} T(t)=T(t) P_{U}$, for all $t \geq 0$.

(ii) $(T(t))_{t \geq 0}$ is a stable $C_{0}$-semigroup on $X^{S}=P_{S} X$ with generator $P_{S} A$, and $(T(t))_{t \in \mathbb{R}}$ is a $C_{0}$-group on $X^{U}=P_{U} X$ with generator $P_{U} A$.

(iii) $P_{S}$ and $P_{U}$ can be extended on $X_{-1}$ to two unique bounded operators $P_{S,-1}$ and $P_{U,-1}$.

We need also the following fundamental lemma; see [2].

Lemma 2.2. Let $f: \mathbb{R} \longrightarrow F_{A_{-1}}$ be a bounded function. Then, the following assertions hold:

$$
\begin{aligned}
& \int_{-\infty}^{t} T_{-1}(t-s) P_{S,-1} f(s) d s, \int_{t}^{\infty} T_{-1}(t-s) P_{U,-1} f(s) d s \in X \text { for all } t \in \mathbb{R}, \\
& \left\|\int_{-\infty}^{t} T_{-1}(t-s) P_{S,-1} f(s) d s\right\| \leq C e^{-\delta t} \int_{-\infty}^{t} e^{\delta s}\|f(s)\|_{F_{A_{-1}}} d s, \\
& \left\|\int_{t}^{+\infty} T_{-1}(t-s) P_{U,-1} f(s) d s\right\| \leq C e^{\delta t} \int_{t}^{+\infty} e^{-\delta s}\|f(s)\|_{F_{A_{-1}}} d s \quad \text { for all } t \in \mathbb{R} .
\end{aligned}
$$

We end this section by recalling the definition of almost automorphic functions and some of their properties.

Definition 2.3 (S. Bochner). A continuous function $f: \mathbb{R} \rightarrow X$ is called almost automorphic if for every sequence $\left(\sigma_{n}\right)_{n \in \mathbb{N}}$ there exists a subsequence $\left(s_{n}\right)_{n \in \mathbb{N}} \subset$ $\left(\sigma_{n}\right)_{n \in \mathbb{N}}$ such that

$$
\lim _{n, m \rightarrow+\infty} f\left(t+s_{n}-s_{m}\right)=f(t) \quad \text { for each } t \in \mathbb{R} .
$$

This is equivalent to

$$
g(t):=\lim _{n \rightarrow+\infty} f\left(t+s_{n}\right) \text { and } f(t)=\lim _{n \rightarrow+\infty} g\left(t-s_{n}\right)
$$

are well defined for each $t \in \mathbb{R}$.

The function $g$ in the definition above is measurable, but not necessarily continuous. Clearly, if the convergence in the definition above is uniform in $t \in \mathbb{R}$, then $f \in A P(X)$, the space of all almost periodic functions with values in $X$. That is, $A P(X) \subset A A(X)$.

It is well known that the range $\mathcal{R}_{f}=f(\mathbb{R})$ of an almost automorphic function $f: \mathbb{R} \rightarrow X$ is relatively compact in $X$, thus it is bounded in norm. Also, the collection $A A(X)$ of all almost automorphic $X$-valued functions is a Banach space under the supnorm $\|f\|_{A A(X)}=\sup _{t \in \mathbb{R}}\|f(t)\|$.

Remark 2.4. An almost automorphic function may not be uniformly continuous.

Example 2.5 (Levitan). Let $p(t)=2+\cos t+\cos \sqrt{2} t$ and $f: \mathbb{R} \rightarrow \mathbb{R}$ such that $f=\sin \frac{1}{p}$. Then $f \in A A(X)$, but $f$ is not uniformly continuous on $\mathbb{R}$. It follows that $f \notin A P(X)$.

Since almost periodic functions (in Bochner's sense) are uniformly continuous, the above remark is very important and indicates that many results and methods in the theory of almost periodicity may not stand in almost automorphy framework. For a complete background on almost automorphic functions one can see [18, 20], and the important Memoirs 22 for almost automorphic dynamics. 


\section{Main abstract Results}

Consider a generator $A$ of a hyperbolic $C_{0^{-}}$-semigroup $(T(t))_{t \geq 0}$ on a Banach space $X$, and the semilinear evolution equation

$$
(S E E) \quad x^{\prime}(t)=A_{-1} x(t)+f(t, x(t)), \quad t \in \mathbb{R} .
$$

The function $f: \mathbb{R} \times X \longrightarrow F_{A_{-1}}$ is continuous and globally Lipschitzian, i.e., there is $k>0$ such that

$$
\|f(t, x)-f(t, y)\|_{F_{A_{-1}}} \leq k\|x-y\| \text { for all } t \in \mathbb{R} \text { and } x, y \in X .
$$

By a mild solution of $(S E E)$ we will understand a continuous function $x: \mathbb{R} \longrightarrow$ $X$, which satisfies the following variation of constants formula

$$
x(t)=T(t-s) x(s)+\int_{s}^{t} T_{-1}(t-\tau) f(\tau, x(\tau)) d \tau \text { for all } t \geq s, t, s \in \mathbb{R} .
$$

We study first the existence of almost automorphic mild solutions for the inhomogeneous evolution equation

$$
(I E E) \quad x^{\prime}(t)=A_{-1} x(t)+g(t), \quad t \in \mathbb{R} .
$$

We have the following main result.

Theorem 3.1. Let $g \in A A\left(F_{A_{-1}}\right)$. Then, the equation (IEE) admits a unique mild solution $x \in A A(X)$ given by

$$
x(t)=\int_{-\infty}^{t} T_{-1}(t-s) P_{S,-1} g(s) d s-\int_{t}^{+\infty} T_{-1}(t-s) P_{U,-1} g(s) d s, \quad t \in \mathbb{R} .
$$

Proof. Let $x(\cdot)$ be the function defined for all $t \in \mathbb{R}$ by

$$
x(t)=\int_{-\infty}^{t} P_{S,-1} T_{-1}(t-s) g(s) d s-\int_{t}^{+\infty} P_{U,-1} T_{-1}(t-s) g(s) d s .
$$

By Lemma 2.2, $x(\cdot)$ is a bounded continuous function from $\mathbb{R}$ to $X$. Moreover, one can see easily that $x(\cdot)$ satisfies the variation of constants formula

$$
x(t)=T(t-s) x(s)+\int_{s}^{t} T_{-1}(t-\tau) g(\tau) d \tau \text { for all } t \geq s, t, s \in \mathbb{R},
$$

that is, $x(\cdot)$ is a bounded mild solution of $(I E E)$. To show the uniqueness, let $u$ be a bounded continuous function $\mathbb{R} \longrightarrow X$ satisfying

$$
u(t)=T(t-s) u(s)+\int_{s}^{t} T_{-1}(t-\tau) g(\tau) d \tau \text { for all } t \geq s, t, s \in \mathbb{R} .
$$

Then, from Proposition 2.1, we get

$$
\begin{array}{ll}
P_{S} u(t)=T(t-s) P_{S} u(s)+\int_{s}^{t} T_{-1}(t-\tau) P_{S,-1} g(\tau) d \tau & \text { for } t \geq s, t, s \in \mathbb{R}, \\
P_{U} u(t)=T(t-s) P_{U} u(s)+\int_{s}^{t} T_{-1}(t-\tau) P_{U,-1} g(\tau) d \tau & \text { for } t, s \in \mathbb{R} .
\end{array}
$$


Since $u$ is bounded, then, by using the estimates (2.2), Lemma 2.2 and by letting $s \rightarrow-\infty$ in (3.4) and $s \rightarrow+\infty$ in (3.5), we obtain

$$
\begin{aligned}
& P_{S} u(t)=\int_{-\infty}^{t} T_{-1}(t-s) P_{S,-1} g(s) d s, \\
& P_{U} u(t)=\int_{+\infty}^{t} T_{-1}(t-s) P_{U,-1} g(s) d s, \quad t \in \mathbb{R} .
\end{aligned}
$$

Consequently, by the decomposition of the space $X$, we obtain that $u(t)=x(t)$, and the uniqueness is proved. To show that the mild solution $x$ is almost automorphic, let $\left(s_{n}^{\prime}\right)$ be an arbitrary sequence of real numbers; then it has a subsequence $\left(s_{n}\right)$ such that $\lim _{n, m} g\left(t+s_{n}-s_{m}\right)=g(t)$ in $F_{A_{-1}}$ for each $t \in \mathbb{R}$, since $g \in A A\left(F_{A_{-1}}\right)$. From Lemma 2.2 we have

$$
\begin{aligned}
& \left\|\int_{-\infty}^{t+s_{n}-s_{m}} T_{-1}\left(t+s_{n}-s_{m}-s\right) P_{S,-1} g(s) d s-\int_{-\infty}^{t} T_{-1}(t-s) P_{S,-1} g(s) d s\right\| \\
& \leq C \int_{-\infty}^{0} e^{\delta s}\left\|g\left(t+s+s_{n}-s_{m}\right)-g(t+s)\right\|_{F_{A_{-1}}} d s
\end{aligned}
$$

and

$$
\begin{aligned}
& \left\|\int_{t+s_{n}-s_{m}}^{+\infty} T_{-1}\left(t+s_{n}-s_{m}-s\right) P_{U,-1} g(s) d s-\int_{t}^{+\infty} T_{-1}(t-s) P_{U,-1} g(s) d s\right\| \\
& \leq C \int_{0}^{+\infty} e^{-\delta s}\left\|g\left(t+s+s_{n}-s_{m}\right)-g(t+s)\right\|_{F_{A_{-1}}} d s .
\end{aligned}
$$

Therefore, $\lim _{n, m \rightarrow \infty}\left\|x\left(t+s_{n}-s_{m}\right)-x(t)\right\|=0$ for each $t \in \mathbb{R}$.

Now, we come back to study the asymptotic behavior of $(S E E)$. To this end, let $A A(\mathbb{R} \times Y, X)$, for some Banach spaces $X$ and $Y$, denote the set of continuous functions $f: \mathbb{R} \times Y \longrightarrow X$ such that $f(\cdot, y) \in A A(X)$ for each $y \in Y$.

Consider now $y \in A A(X)$ and $f \in A A\left(\mathbb{R} \times X, F_{A_{-1}}\right)$. Then, by 20, Theorem 2.2.4], the function $g(\cdot):=f(\cdot, y(\cdot)) \in A A\left(F_{A_{-1}}\right)$ and from Theorem 3.1, the inhomogeneous evolution equation

$$
x^{\prime}(t)=A x(t)+g(t), \quad t \in \mathbb{R},
$$

admits a unique mild solution $x \in A A(X)$ given by

$$
x(t)=\int_{-\infty}^{t} T_{-1}(t-s) P_{S,-1} f(s, y(s)) d s-\int_{t}^{+\infty} T_{-1}(t-s) P_{U,-1} f(s, y(s)) d s .
$$

Let the operator $F: A A(X) \longrightarrow A A(X)$ be defined by

$$
\begin{aligned}
(F y)(t) & : \int_{-\infty}^{t} T_{-1}(t-s) P_{S,-1} f(s, y(s)) d s \\
& -\int_{t}^{+\infty} T_{-1}(t-s) P_{U,-1} f(s, y(s)) d s \quad \text { for all } t \in \mathbb{R}
\end{aligned}
$$


and assume that $k C<\frac{\delta}{2}$, where $C$ is the constant defined in Lemma 2.2. Then, we have for any $x, y \in A A(X)$,

$$
\begin{aligned}
\|F x(t)-F y(t)\| \leq & C e^{-\delta t} \int_{-\infty}^{t} e^{\delta s}\|f(s, x(s))-f(s, y(s))\|_{F_{A_{-1}}} d s \\
& +C e^{\delta t} \int_{t}^{+\infty} e^{-\delta s}\|f(s, x(s))-f(s, y(s))\|_{F_{A_{-1}}} d s . \\
\leq & \frac{2 k C}{\delta}\|x-y\|_{\infty} \text { for all } t \in \mathbb{R} .
\end{aligned}
$$

This shows that $F$ has a unique fixed point in $A A(X)$, and consequently we have the following theorem.

Theorem 3.2. Assume that $k C<\frac{\delta}{2}$ and $f \in A A\left(\mathbb{R} \times X, F_{A_{-1}}\right)$. Then $(S E E)$ admits a unique mild solution $x$ in $A A(X)$, which satisfies the variation of constants formula

$x(t)=\int_{-\infty}^{t} T_{-1}(t-s) P_{S,-1} f(s, x(s)) d s-\int_{t}^{+\infty} T_{-1}(t-s) P_{U,-1} f(s, x(s)) d s, t \in \mathbb{R}$.

\section{Semilinear boundary DifFerential EQUATions}

Consider the semilinear boundary differential equation

$$
\left\{\begin{array}{l}
x^{\prime}(t)=A_{m} x(t)+h(t, x(t)), \quad t \in \mathbb{R} \\
L x(t)=g(t, x(t)), \quad t \in \mathbb{R}
\end{array}\right.
$$

Here $\left(A_{m}, D\left(A_{m}\right)\right)$ is a densely defined linear operator on a Banach space $X, L$ : $D\left(A_{m}\right) \longrightarrow \partial X$, the boundary Banach space and the functions $h: \mathbb{R} \times X \longrightarrow X$, $g: \mathbb{R} \times X \longrightarrow \partial X$ are continuous.

We shall make the following assumptions introduced by G. Greiner [1].

(A1) There exists a new norm $|\cdot|$ which makes the domain $D\left(A_{m}\right)$ complete and then denoted by $X_{m}$. The space $X_{m}$ is continuously embedded in $X$ and $A_{m} \in \mathcal{L}\left(X_{m}, X\right)$.

(A2) The restriction $A:=\left.A_{m}\right|_{\operatorname{ker}(L)}$ generates a $C_{0}$-semigroup $T(\cdot)$ on $X$.

(A3) $L \in \mathcal{L}\left(X_{m}, \partial X\right)$ is surjective.

(A4) There exist positive constants $\gamma, \lambda_{0}$ such that

$$
\|L x\| \geq \gamma\left(\lambda-\lambda_{0}\right), \quad x \in \operatorname{ker}\left(\lambda-A_{m}\right), \lambda \in \rho(A), \lambda>\lambda_{0} .
$$

(A5) The semigroup $T(\cdot)$ is hyperbolic on $X$.

One can see that under (A1)-(A2) and for some $\lambda \in \rho(A)$ the maximal domain $X_{m}$ can be decomposed as

$$
X_{m}=\operatorname{Ker} L \oplus \operatorname{ker}\left(\lambda-A_{m}\right) ;
$$

see [11. Thus, the restriction $L: \operatorname{ker}\left(\lambda-A_{m}\right) \rightarrow \partial X$ is then a bijection and its inverse is the so-called Dirichlet operator $L_{\lambda} \in \mathcal{L}(\partial X, X)$ and $L_{\lambda} L$ is a projection onto $\operatorname{ker}\left(\lambda-A_{m}\right)$. From (4.2), one can show also that

$$
x \in D\left(A_{m}\right) \Longleftrightarrow x-L_{\lambda} L x \in D(A) .
$$

It is shown also in [1] that

$$
R(\mu, A) L_{\lambda}=R(\lambda, A) L_{\mu} \quad \text { for all } \lambda, \mu \in \rho(A) .
$$


We know also from [7] that the assumption (A4) is equivalent to the fact that the operator

$$
L_{\lambda}: \partial X \longrightarrow F_{A} \text { is bounded for all } \lambda>\lambda_{0} .
$$

Recall here that $x: \mathbb{R} \longrightarrow X$ is a mild solution of (SBDE) if for all $t \geq s, t, s \in \mathbb{R}$, we have

$$
\begin{aligned}
& \text { (i) } \int_{s}^{t} x(\tau) d \tau \in X_{m}, \\
& \text { (ii) } x(t)-x(s)=A_{m} \int_{s}^{t} x(\tau) d \tau+\int_{s}^{t} h(\tau, x(\tau)) d \tau, \\
& \text { (iii) } L \int_{s}^{t} x(\tau) d \tau=\int_{s}^{t} g(\tau, x(\tau)) d \tau .
\end{aligned}
$$

In the following lemma we show the equivalence between the boundary equation (SBDE) and an evolution equation.

Lemma 4.1. Assume that (A1)-(A3) are satisfied. A function $x$ is a mild solution of the boundary equation (SBDE) if and only if $x$ is a mild solution of the semilinear evolution equation on $X$,

$$
(S E E) \quad x^{\prime}(t)=A_{-1} x(t)+h(t, x(t))-A_{-1} L_{0} g(t, x(t)), \quad t \in \mathbb{R} .
$$

Proof. Let $x$ be a mild solution of (SBDE). Then, since $\operatorname{Range}\left(L_{0}\right) \subset \operatorname{ker}\left(A_{m}\right)$ and from (4.3), we have

$$
\begin{aligned}
x(t)-x(s)= & A_{m} \int_{s}^{t} x(\tau) d \tau+A_{m} L_{0} L \int_{s}^{t} x(\tau) d \tau+\int_{s}^{t} h(\tau, x(\tau)) d \tau \\
= & A\left(\int_{s}^{t} x(\tau) d \tau-L_{0} L \int_{s}^{t} x(\tau) d \tau\right)+\int_{s}^{t} h(\tau, x(\tau)) d \tau \\
= & A_{-1} \int_{s}^{t} x(\tau) d \tau+\int_{s}^{t} h(\tau, x(\tau)) d \tau \\
& -A_{-1} L_{0} \int_{s}^{t} g(\tau, x(\tau)) d \tau, \quad t \geq s, t, s \in \mathbb{R} .
\end{aligned}
$$

The last equation is equivalent to the fact that $x$ satisfies the variation of constants formula (3.3), and then is a mild solution of (SEE). Now let $x$ be a mild solution of (SEE), that is, $x$ satisfies

$$
\begin{aligned}
x(t)= & T(t-s) x(s)+\int_{s}^{t} T(t-\tau) h(\tau, x(\tau)) d \tau \\
& -\int_{s}^{t} T_{-1}(t-\tau) A_{-1} L_{0} g(\tau, x(\tau)) d \tau \quad \text { for all } t \geq s, t, s \in \mathbb{R} .
\end{aligned}
$$

Since $x$ is an $X$-valued function, then $\int_{s}^{t} T_{-1}(t-\tau) A_{-1} L_{0} g(\tau, x(\tau)) d \tau \in X$, and then $\int_{s}^{t} T(t-\tau) L_{0} g(\tau, x(\tau)) d \tau \in D(A)$, and

$$
x(t)=T(t-s) x(s)+\int_{s}^{t} T(t-\tau) h(\tau, x(\tau)) d \tau-A \int_{s}^{t} T(t-\tau) L_{0} g(\tau, x(\tau)) d \tau .
$$


Hence,

$$
\begin{aligned}
& \int_{s}^{t} x(\tau) d \tau \\
& =\int_{s}^{t} T(\tau-s) x(s) d \tau+\int_{s}^{t} \int_{s}^{\tau} T(\tau-\sigma) h(\sigma, x(\sigma)) d \sigma d \tau \\
& \quad-\int_{s}^{t} \int_{s}^{\tau} T_{-1}(\tau-\sigma) A_{-1} L_{0} g(\sigma, x(\sigma)) d \sigma d \tau \\
& =A^{-1}[T(t-s) x(s)-x(s)]+A^{-1} \int_{s}^{t} T(t-\sigma) h(\sigma, x(\sigma)) d \sigma+A^{-1} \int_{s}^{t} h(\sigma, x(\sigma)) d \sigma \\
& -\int_{s}^{t} T(t-\sigma) L_{0} g(\sigma, x(\sigma)) d \sigma-L_{0} \int_{s}^{t} g(\sigma, x(\sigma)) d \sigma .
\end{aligned}
$$

This yields easily that $x$ satisfies (i)-(iii) above. This achieves the proof.

We can now announce the main result of this section.

Theorem 4.2. Assume that (A1)-(A5) are satisfied, and that the functions $g \in$ $A A(\mathbb{R} \times X, \partial X), h \in A A(\mathbb{R} \times X, X)$ are globally Lipschitzian with small constants. Then the semilinear boundary differential equation $(S B D E)$ has a unique almost automorphic mild solution $x$ satisfying, for all $t \in \mathbb{R}$,

$$
\begin{aligned}
& x(t)=\int_{-\infty}^{t} T(t-s) P_{S} h(s, x(s)) d s-\int_{t}^{+\infty} T(t-s) P_{U} h(s, x(s)) d s \\
& 4.6) \quad-A\left[\int_{-\infty}^{t} T(t-s) P_{S} L_{0} g(s, x(s)) d s-\int_{t}^{+\infty} T(t-s) P_{U} L_{0} g(s, x(s)) d s\right] .
\end{aligned}
$$

Proof. From equations (2.1), (4.4) and (4.5), $A_{-1} L_{0}$ is a bounded operator from $\partial X$ to $F_{A_{-1}}$. Hence, since $g \in A A(\mathbb{R} \times X, \partial X)$ and $h \in A A(\mathbb{R} \times X, X)$ and from the injection $X \hookrightarrow F_{A_{-1}}$, the function $f(t, x):=h(t, x)-A_{-1} L_{0} g(t, x)$ belongs to $A A\left(\mathbb{R} \times X, F_{A_{-1}}\right)$. This function is also globally Lipschitzian with a small constant. Hence, by Theorem 3.2 there is a unique mild solution $x \in A A(X)$ of the equation (SEE), satisfying

$$
x(t)=\int_{-\infty}^{t} P_{S,-1} T_{-1}(t-s) f(s, x(s)) d s-\int_{t}^{+\infty} P_{U,-1} T_{-1}(t-s) f(s, x(s)) d s,
$$

from which we deduce the variation of constants formula (4.6). The above lemma yields that $x$ is the unique almost automorphic mild solution of (SBDE).

\section{RETARDED DiffERENTIAL EQUATIONS}

Consider the semilinear retarded differential equation

$$
(R D E) \quad \frac{d}{d t} x(t)=B x(t)+g\left(t, x_{t}\right), \quad t \in \mathbb{R},
$$

where $B$ generates an immediately compact or norm continuous $C_{0}$-semigroup $(S(t))_{t \geq 0}$ on a Banach space $E, g$ is a function from $\mathbb{R} \times C([-r, 0], E)$ into $E$. 
This equation can be written as a boundary differential equation, by setting

$$
\begin{gathered}
X=C([-r, 0], E), \quad \partial X=E, \quad A_{m}=\frac{d}{d \sigma}, \\
D\left(A_{m}\right)=\left\{f \in C^{1}([-r, 0], E): f(0) \in D(B)\right\}, \\
X_{m}=\left(D\left(A_{m}\right),|\cdot|\right), \quad|f|=\|f\|_{\infty}+\left\|f^{\prime}\right\|_{\infty}+\|B f(0)\|, \quad f \in D\left(A_{m}\right) .
\end{gathered}
$$

From the closedness of $B,\left(X_{m},|\cdot|\right)$ is a Banach space, and it is continuously embedded in $X$, and hence (A1) is satisfied. The boundary operator $L$ is defined on $X_{m}$ by $L f=f^{\prime}(0)-B f(0), f \in X_{m}$. It is clear that $L: X_{m} \longrightarrow E$ is bounded and surjective, and $L_{\lambda} x=e_{\lambda} R(\lambda, B) x$ for $x \in E$ and $\lambda \in \rho(B)$, where $e_{\lambda}(\theta)=e^{\lambda \theta}$ for $\theta \in[-r, 0]$. The assumptions (A3) and (A4) are then satisfied. The operator $A=A_{m} \mid \operatorname{ker} L$ is given by

$$
A=\frac{d}{d \sigma}, \quad D(A)=\left\{f \in C^{1}([-r, 0], E): f(0) \in D(B), f^{\prime}(0)=B f(0)\right\}
$$

and it generates a $C_{0}$-semigroup $(T(t))_{t \geq 0}$ on $X$; see for instance, [9, Theorem 6.1] and [23. This yields the assumption (A)2). From [9, Thm. 6.6, Thm. 6.9], this semigroup is eventually (for $t>r$ ) compact (resp. norm continuous) if $(S(t))_{t \geq 0}$ is immediately compact (resp. norm continuous).

We have also, from [9, Proposition 6.7], that $\sigma(A)=\sigma(B)$. If we assume now that $\sigma(B) \cap i \mathbb{R}=\emptyset$, then the semigroup $(T(t))_{t \geq 0}$ is hyperbolic.

Theorem 5.1. Assume $\sigma(B) \cap i \mathbb{R}=\emptyset$. If $g \in A A(\mathbb{R} \times C([-r, 0], E), E)$ and globally Lipschitzian with a small constant, then $(R D E)$ admits a unique mild solution $x$ such that $\mathbb{R} \ni t \longmapsto x_{t}$ is almost automorphic in $C([-r, 0], E)$ and satisfies

$$
\begin{aligned}
x_{t}=-A\left[\int_{-\infty}^{t} T(t-s) P_{S} e_{0} B^{-1} g\left(s, x_{s}\right) d s\right. & \\
& \left.-\int_{t}^{+\infty} T(t-s) P_{U} e_{0} B^{-1} g\left(s, x_{s}\right) d s\right], t \in \mathbb{R} .
\end{aligned}
$$

Example. Consider the following retarded partial differential equation:

$$
\left\{\begin{array}{l}
\frac{\partial}{\partial t} u(t, x)=\frac{\partial^{2}}{\partial x^{2}} u(t, x)+\alpha u(t, x)+g(t, u(t-1, x)), t \in \mathbb{R}, x \in[0, \pi], \\
u(t, 0)=u(t, \pi)=0, t \in \mathbb{R}
\end{array}\right.
$$

where $g: \mathbb{R} \times \mathbb{R} \longrightarrow \mathbb{R}$ such that for all $t \in \mathbb{R}, f(t, u(t-1, \cdot)) \in L^{2}(0, \pi)$ and $\alpha \in \mathbb{R}$.

Let $E:=L^{2}(0, \pi)$, the equation (5.1) can be written in $E$ as the following retarded differential equation:

$$
\frac{d}{d t} v(t)=B v(t)+g\left(t, v_{t}\right), t \in \mathbb{R},
$$

with $v, g: \mathbb{R} \rightarrow E$ such that $v(t)=u(t, \cdot)$ and $g(t, \varphi)=g(t, \varphi(-1, \cdot)), v_{t} \in$ $C([-1,0], E)$, and $B$ is the operator defined in $E$ by

$$
B y=y^{\prime \prime}+\alpha y, y \in D(B)=\left\{y \in W^{2,2}([0, \pi]) ; y(0)=y(\pi)=0\right\} .
$$

It is well known that the operator $B$ generates an immediately compact semigroup in $E$, and that $\lambda \in \sigma(B)$ if and only if there exists $n \in \mathbb{N}$ such that

$$
\left(E_{n}\right) \quad \lambda=\alpha-n^{2} .
$$


If we suppose, for instance, $3<\alpha<4$, then all solutions of the equations $\left(E_{n}\right)$ are in $\mathbf{C} \backslash i \mathbb{R}$. Therefore, $\left(E_{1}\right)$ admits a positive real solution. We deduce that the type of $B$ is not negative and $\sigma(B) \cap i \mathbb{R}=\emptyset$. Then all assumptions of Theorem 5.1 are satisfied and thus one has the same conclusion for the retarded differential equation (5.1).

\section{ACKNOWLEDGEMENT}

The authors of this paper would like to thank the referee for his/her useful comments and suggestions.

\section{REFERENCES}

[1] H. Amann, Linear and Quasilinear Parabolic Problems. Birkhäuser, Berlin 1995. MR.1345385 (96g:34088)

[2] B. Amir and L. Maniar, Existence and some asymptotic behaviors of solutions to semilinear Cauchy problems with non dense domain via extrapolation spaces, Rend. Circ. Mat. Palermo (2000) 481-496. MR:1809089 (2001m:35190)

[3] B. Amir and L. Maniar, Composition of pseudo-almost periodic functions and Cauchy problems for Hille-Yosida operators, Ann. Math. Blaise Pascal 6 (1999), 1-11. MR.1693142 (2000h:34094)

[4] A. Bátkai, L. Maniar and A. Rhandi, Regularity properties of perturbed Hille-Yosida operators and retarded differential equations, Semigroup Forum 64 (2002), 55-70. MR.1866316 (2002h:47060)

[5] S. Boulite, L. Maniar and G. M. N'Guérékata, Almost automorphic solutions for hyperbolic semilinear evolution equations, Semigroup Forum, 71 (2005), 231-240. MR2184055

[6] G. Da Prato and P. Grisvard, On extrapolation spaces, Rend. Accad. Naz. Lincei. 72 (1982), 330-332. MR0726298 (85j:46131)

[7] W. Desch, W. Schappacher and K.P. Zhang, Semilinear evolution equations, Houston J. Math. 15 (1989), 527-552. MR1045511 (92d:47087)

[8] T. Diagana, G. N'Guérékata and N. Van Minh, Almost automorphic solutions of evolution equations, Proc. Amer. Math. Soc., 132 (2004), 3289-3298. MR2073304 (2005d:34124)

[9] K. J. Engel and R. Nagel, One-Parameter Semigroups for Linear Evolution Equations, Graduate Texts in Mathematics, Springer-Verlag 1999. MR1721989 (2000i:47075)

[10] J. A. Goldstein, G. M. N'Guérékata, Almost automorphic solutions of semilinear evolution equations, Proc. Amer. Math. Soc. 133 (2005), 2401-2408. MR2138883 (2006a:34175)

[11] G. Greiner, Perturbing the boundary conditions of a generator, Houston J. Math. 13 (1987), 213-229. MR0904952 (88i:47023)

[12] E. Hernández, M. Pelicer and J. dos Santos, Asymptotically almost periodic and almost periodic solutions for a class of evolution equations, Elect. J. Diff. Equat. (2004), 1-15. MR2047417 (2004m:34164)

[13] Y. Hino, S. Murakami, Almost automorphic solutions for abstract functional differential equations, J. Math. Math. Analysis and Appl. 286 (2003), 741-752. MR2008862 (2004i:34160)

[14] R. A. Johnson, A linear almost periodic equation with an almost automorphic solution, Proc. Amer. Math. Soc., Vol. 82, No. 2 (1981), 199-205. MR0609651 (82i:34044a)

[15] L. Maniar and A. Rhandi, Inhomogeneous retarded Differential equation in infinite dimensional Banach space via extrapolation spaces, Rend. Circ. Mat. Palermo 47 (1998), 331-346. MR.1633503 (99f:34116)

[16] L. Maniar, Théorie de Perturbations et Propriétés Qualitatives de $C_{0}$-semi-groupes, Thesis, Marrakesh, 2001.

[17] R. Nagel and E. Sinestrari, Inhomogeneous Volterra integrodifferential equations for HilleYosida operators. Marcel Dekker, Lecture Notes Pure Appl. Math. 150 (1994). MR1241671 (94i:34121)

[18] G. M. N'Guérékata, Almost Automorphic Functions and Almost Periodic Functions in Abstract Spaces, Kluwer Academic/Plenum Publishers, New York, 2001. MR1880351 (2003d:43001) 
[19] G. M. N'Guérékata, Existence and uniqueness of almost automorphic mild solution to some semilinear abstract differential equations, Semigroup Forum 69 (2004), 80-86. MR2063980 (2005b:34119)

[20] G. M. N'Guérékata, Topics in Almost Automorphy, Springer, New York, Boston, Dordrecht, London, Moscow, 2005. MR2107829 (2005h:34153)

[21] G. Nickel and A. Rhandi, On the essential spectral radius of semigroups generated by perturbations of Hille-Yosida operators, J. Diff. Integ. Equat., to appear.

[22] W. Shen and Y. Yi, Almost Automorphic and Almost Periodic Dynamics in Skew-Product Semiflows, Memoirs Amer. Math. Soc., 647, Vol. 136 (1998). MR1445493 (99d:34088)

[23] J. Wu, Theory and Applications of Partial Functional Differential Equations, App. Math. Sci. 119, 1996. MR.1415838(98a:35135)

Department of Mathematics, Faculty of Sciences Semlalia, B.P. 2390, Marrakesh, MOROCCO

E-mail address: sboulite@ucam.ac.ma

Department of Mathematics, Faculty of Sciences Semlalia, B.P. 2390, Marrakesh, MOROCCO

E-mail address: maniar@ucam.ac.ma

Department of Mathematics, Morgan State University, 1700 E. Cold Spring Lane, Baltimore, Maryland 21251

E-mail address: gnguerek@morgan.edu. 